\title{
On the Origin of the Two Way Shape Memory Effect in Cu-Zn-Al Alloys
}

\author{
F.C. Lovey, P.L. Rodriguez, J. Malarria, M. Sade and V. Torra* \\ Centro Atómico Bariloche,(Comisión Nacional de Energía Atómica), 8400 S.C. de Bariloche, Argentina \\ * Departament de Física Aplicada, Universitat Politécnica de Catalunya, E-08034 Barcelona, Spain
}

\begin{abstract}
Several training procedures have been found suitable to induce the two way shape memory effect (TWSME) in copper based alloys. Complex dislocation arrangements can be produced by transformation cycling, which are believed to be the cause of the TWSME. Small martensite plates can be stabilized, serving as origin of subsequent transformations, or deformation around second phase precipitates are used. In this work, the role of the dislocations arrangements is investigated. It is shown that the stress field around the dislocations leads to the formation, in the $\beta$ phase, above the Ms, of small martensite plates. These martensites do not necessarily correspond to the trained variant. Complex defects consisting of dislocations and heavily deformed martensites are thus produced. The role of these complex defects on the TWSME is discussed and possible mechanisms are suggested.
\end{abstract}

\section{INTRODUCTION}

The two way shape memory effect (TWSME) has been a subject of increasing interest during the last years. Several methods have been found effective to induce the TWSME: thermomechanical training [1,2], constraint aging [3] or by producing small plates of stabilized martensite [4]. In addition, the presence of precipitates of a second phase usually enhances the TWSME [5]. Among them, the mechanical or thermomechanical cycling has been more widely used to study such effect in $\mathrm{Cu}-\mathrm{Zn}-\mathrm{Al}$ alloys [6-9]. Recently, it has been shown that the TWSME is in fact a stable effect and can deliver work on cooling $[5,7,9]$. This has been observed in pseudoelastically cycled single crystals either free of precipitates [9] or containing $\gamma$ precipitates [5], and in thermomechanically trained polycrystals [7]. In the materials free of precipitates, the training procedures give rise to complex dislocation arrays $[1,8,10,11]$. Based on these observations the TWSME is believed to be related to such dislocations. The changes of the dislocation energies in different variants of martensite have been analyzed $[8,12]$ as a possible factor making one variant more stable. This could be more important if dislocation arrays are considered [7]. On the other hand, it was suggested that the residual stress due to the dislocations would only favor the nucleation and the beginning of the growth of preferential variants $[1,10]$. More recently it has been mentioned that an other factor can contribute to the TWSME [13]. This is related to the martensite stabilization during cycling. Not complete recovery of the $\beta$ phase would occur, leaving some degree of disordering favoring the trained variant.

Thus, several mechanisms could be responsible of the TWSME, none of them are well understood yet. Some new evidence of the defects formed during the training and the possible relation with the TWSME are presented and discussed in this work. 


\section{EXPERIMENTAL RESULTS}

Single crystals of $\mathrm{Cu}-\mathrm{Zn}-\mathrm{Al}$ alloys, with $\mathrm{Ms}$ temperatures between $273 \mathrm{~K}$ and $283 \mathrm{~K}$, were pseudoelastically cycled at around room temperature. About 3000 cycles were found necessary to induce more than $80 \%$ of TWSME, in agreement with previous results [8].

Optical microscopy observations showed the appearance of surface defects with traces parallel to both the habit plane and the basal plane of the induced martensite variant. Theses defects have been studied in detail before [14]. The habit plane defects can be eliminated by surface polishing and can be disregarded as a cause of the TWSME. On the other hand the defects parallel to the basal plane remain after polishing this has been attributed to the intersection of bulk defects with the surface [14]. The specimens exhibiting the TWSME were also analyzed by transmission electron microscopy in the $\beta$ phase state. Bands of dislocations parallel to either the habit plane or the basal plane were observed, having the burgers vectors $b=[010]_{\beta}$, as described in detail previously [11]. Frequently it was found that the dislocation bands, particularly those parallel to the basal plane contained martensite plates, as shown in Fig. 1. None of the martensite plates observed in Fig. 1 are of the trained variant.

Another interesting defect is shown in Fig. 2. The band parallel to the basal plane contains a dense array of dislocations and heavily deformed martensite of a different variant to the induced one. Nearty perpendicular to the band some clean martensite plates are present. It was confirmed that these plates correspond indeed to the induced variant, as shown in the dark field image and corresponding diffraction patterns of Figs. $2 b$ and $2 c$.

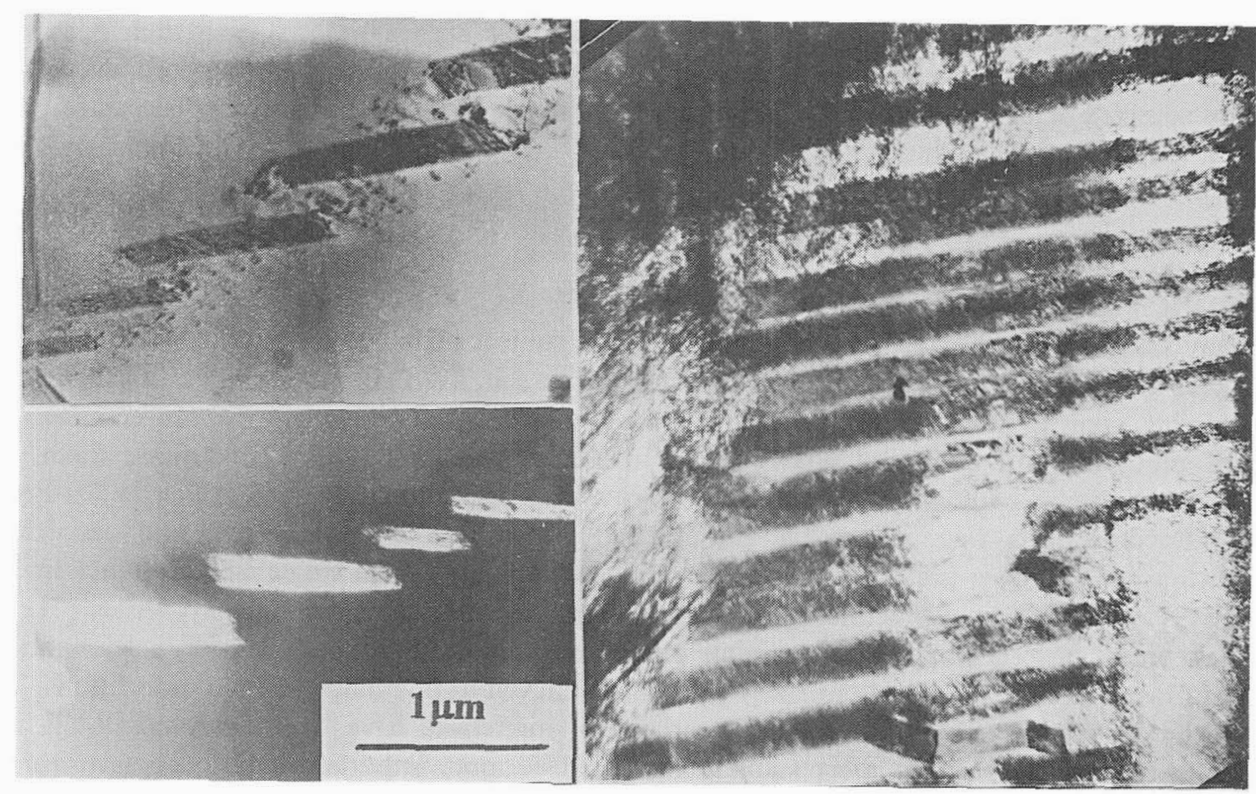

Figure 1: Retained martensite plates in the dislocation bands. None of the plates correspond to the trained variant.

\section{ABOUT THE STRESS FIELD OF THE DISLOCATIONS}

The dislocation arrays are due to localized slip on the martensite basal plane. This can be explained from the lower critical resolved shear stress (CRSS) of the martensite (77 MPa [15]) as compared to the CRSS of the $\beta$ phase ( $200 \mathrm{MPa}$ [16]). For dense dislocations bands the stress field created by the dislocations can be very high in the $\beta$ phase (even higher than $200 \mathrm{MPa}$ ). 

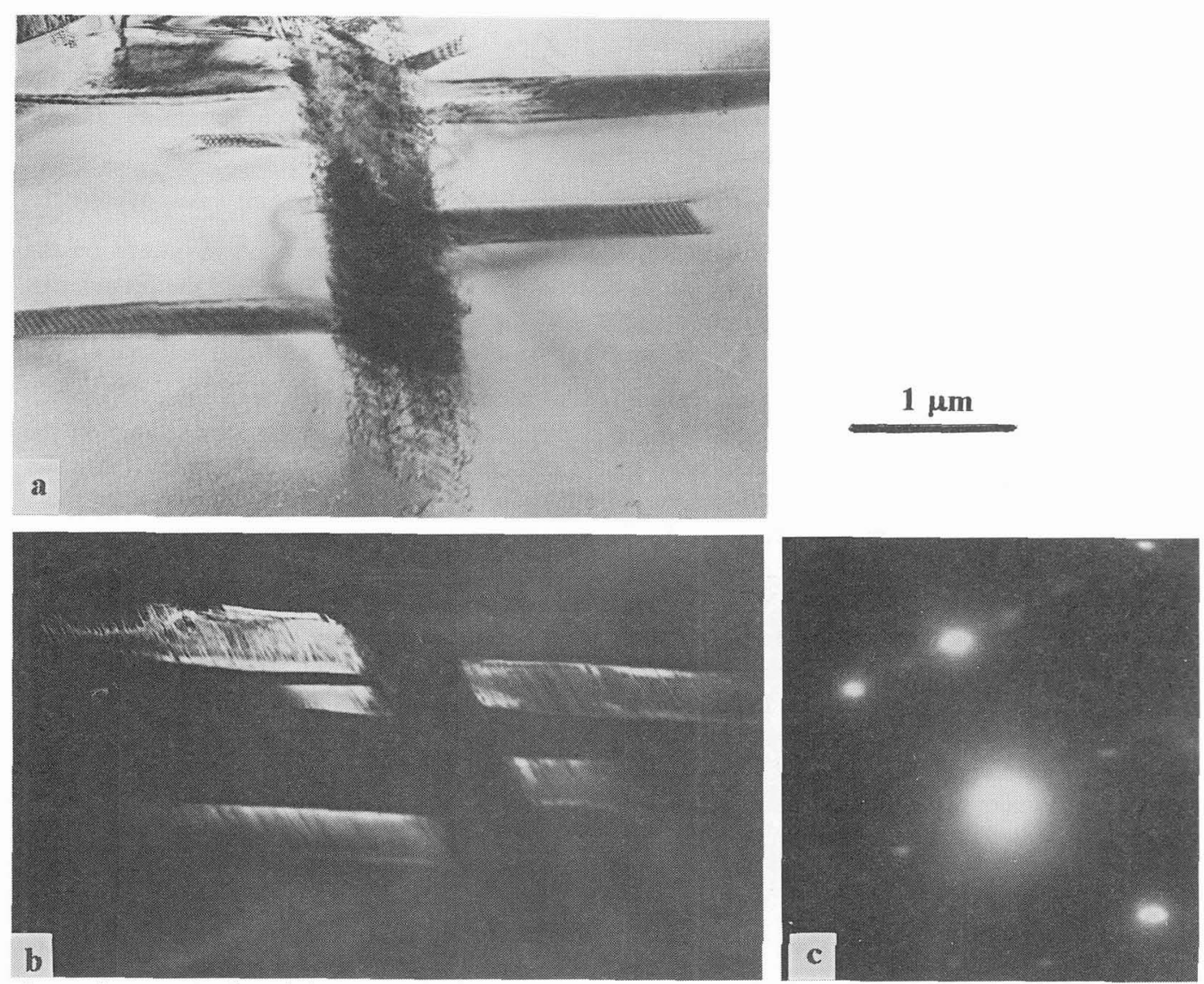

Figure 2: (a) Complex defect of dislocations and heavily deformed martensite (vertical band). Plates of the trained variant (horizontal) remain aside the defect. (b) and (c) are the dark field and diffraction pattern of the trained variant respectively.

The change on the free energy of the material due to the dislocations and a nucleus of martensite with volume $v$, can be evaluated in the classical way using the formulation of Olson and Cohen [17] .

$\Delta G=G_{d}+G_{1}+\Delta g v+E_{a l}+E_{s}$

where $G_{d}$ is the dislocation array self-energy, $\Delta g$ is the free energy difference of the pure phases, $E_{\theta 1}$ and $E_{s}$ are the elastic and surface energies respectively. The interaction energy of the nucleus with the stress field $\sigma_{i j}$, due to the dislocations, is given by the expression of Eshelby [18]:

$$
G_{1}=-\int \sigma_{\mathrm{l}} \varepsilon_{\mathrm{l}} \mathrm{dv}
$$

where $\varepsilon_{i j}$ is the stress free transformation strain. Taken an average value of $50 \mathrm{MPa}$ for the stress field and 0.2 for the shear transformation strain, the interaction energy per volume unit is $E_{1}=-100 \times 10^{5} E_{1}=-100 \times 10^{5} \mathrm{~J} / \mathrm{m}^{3}$. This can be compared with the driving force due to the undercooling, which is giving by: $\Delta \mathrm{G}=\Delta \mathrm{s} \Delta \mathrm{T}$, where $\Delta \mathrm{s}=-2 \times 10^{5} \mathrm{~J} / \mathrm{m}^{3} \mathrm{~K}$, is the entropy change of the transformation per volume unit and $\Delta T=T_{0}-T$ is the amount of undercooling from the equilibrium temperature $T_{0}$. Hence the interaction energy is equivalent to an undercooling of $50 \mathrm{~K}$ in 
the region where the average value of the stress field is $50 \mathrm{MPa}$.

Calculations show that the stress field around the dislocation bands [19] are significant for most of the shear systems of the $<110>\{1 \overline{1} 0\}$ type. Thus several martensite variants can appear around the dislocation bands, as it is indeed experimentally observed ( Fig. 1).

In addition, because of the properties of the elastic stress fields and the constant value of $\varepsilon_{\sharp}$, the interaction energy $G_{1}$ given by the Eshelby formula, would average to zero when integrated on the whole volume of the specimen, i.e. when the whole material has transformed Therefore the presence of stress fields would not make one variant more stable than the others, except for a small percentage restricted to nucleation and initial growth. On the other hand, the self-energy of the dislocations will gradually change from the value $G_{d}^{B}$, when embedded in the pure parent phase, to a value $G_{d}^{m}$ when embedded in martensite. Thus, at the end of the transformation the effect of the dislocations on the stabilities of phases and variants will be reduced to the changes in the dislocation energies. Particular distribution of dislocations arrays could promote the nucleation of the induced variant, however more experimental work along this line is needed.

\section{ABOUT THE DISLOCATION ENERGY}

It has been described in detail elsewhere $[8,12]$ that, given a dislocation with a Burgers vector $b$ in the parent phase, for 16 martensite variants that Burgers vector will transform into a vector with no translation symmetry. Hence, stacking faults will be created increasing the free energy of those variants. Concerning the length of the Burgers vector, it has been shown $[8,12]$ that for 8 of these variants (set $A$ ) the Burgers vector becomes longer by about $10 \%$, while for the other group of 8 variants (set B) the length of the Burgers vector remains almost the same.
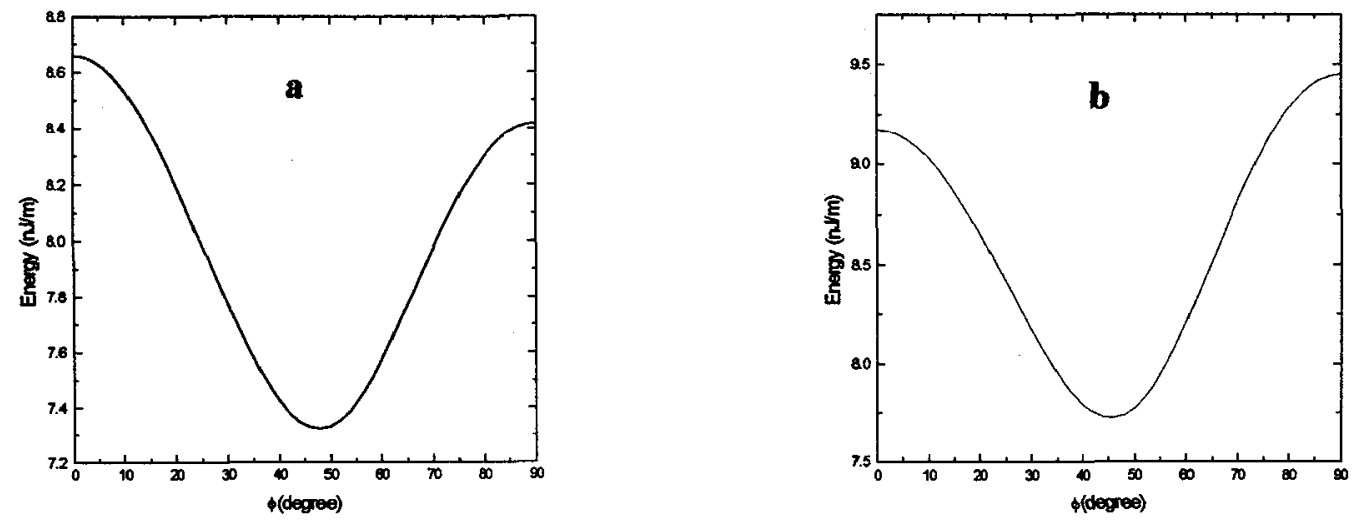

Figure 3: Elastic energy of the dislocations with Burgers vector on the basal plane. Line direction on the basal plane (a), and out of the basal plane (b). $\phi$, the angle between line direction and Burgers vector.

There is still a set of 8 variants (set $\mathrm{C}$ ), where the Burgers vector has translation symmetry; and in addition it becomes shorter. This set of 8 variants can be further divided into two subsets of 4 variants each one. In one of the subsets (subset $C_{1}$ ) the dislocations will glide on the basal plane and for the other subset $\left(C_{2}\right)$ the glide plane will be close to a prismatic type plane [19].

The energies of these two dislocation types have been calculated using the elastic constants of the 18R martensite [20] . Superlattice dislocations have been considered; and in addition Shockley dissociation was allowed for the dislocation gliding on the basal plane. The antiphase boundary and stacking faults energies were taken from ref. [21]. The results are shown in Fig. 3. It is observed in Fig. 3 that the 
dislocations gliding on the basal plane have a slightly lower energy.

The best explanation of the TWSME was suggested to be related to the energy of the dislocations arrays by Stalmans et al. [7]. These authors assumed that the dislocation arrays would have the lowest energy in the trained variant. However such arrays consist essentially in dislocation pile up on the $\{110\}_{\beta}$ plane. These arrays are equivalent for the subsets of 4 variants with basal planes derived from the same $\{110\}_{\beta}$ plane. Therefore, no complete selection between the variants can be expected even if the energy of the dislocation arrangements are considered. Moreover, calculations using the 18R elastic constants and the anisotropic theory of dislocations showed that the energies of dislocation arrangements in the subsets $C_{1}$ and $C_{2}$ have only a small difference [19]. Hence its contribution to the TWSME is expected to be very weak.

It can be concluded in this section that, from the energetic point of view, the dislocations can provide a selection rule for some variants, making to some extent unfavorable the 16 variants of sets $\mathrm{A}$ and $\mathrm{B}$, where the Burgers vectors are longer and, in addition, have not translation symmetry. The other 8 variants of set $\mathrm{C}$ remain nearly equally favorable.

\section{THE ROLE OF THE COMPLEX DEFECTS ON THE TWSME}

The complex defects as those shown in Fig. 1 and 2 behave essentially as an inclusion in the matrix. Such inclusion will be absorbed by the induced variant in a further cycle. This is schematically depicted in Fig. 4. A spherical shape of the inclusion is assumed (Fig. 4a). During the transformation the matrix around the inclusion is deformed in accordance with the macroscopic shear deformation, with a value of about $20 \%$ (Fig. 4 b). The inclusion (and probably its surroundings) is plastically deformed in order to match with the new shape of the cavity (Fig. 4c). In the retransformation the inclusion should go back to the spherical shape through plastic deformation. This latter plastic deformation can be avoided if some plates of the induced variants are left around the inclusion, the deformation is thus elastically absorbed. This is a possible explanation for the presence of the induced variant around the inclusion in Fig. 2. In fact the process described in Fig. 4 occurs gradually during cycling, first dislocations are created and then the inclusion start forming.

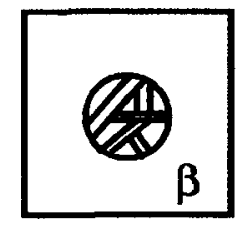

a

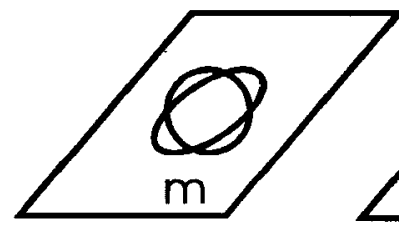

b

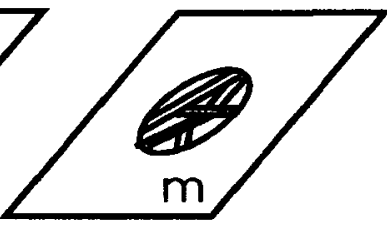

C

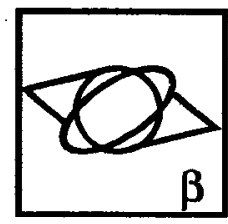

d

Figure 4: The deformation process of the inclusion upon martensitic transformation.

The dislocations and inclusions could favor the TWSME because of the following reasons:

i) The images of Fig. 2 suggests that the stress field around the inclusion would favor the nucleation of the trained variant. Small plates of the trained variant could remain around the inclusions improving the TWSME. The other martensite variants in the inclusion are heavily deformed and subjected to the dislocation attraction, they are not free to grow.

ii) In order to compensate the shape deformation, the initial growth of the trained variant would trigger the appearance of the opposite variant, However, the opposite variant belongs into the group of 16 variants where the dislocation Burgers vector has no translation symmetry. Thus the formation of self 
accommodating groups are impeded by the dislocations, and the growth of the trained variant is favored. iii) The inclusions are pre-deformed according to the trained variant. The elasto-plastic energy to absorb such inclusions would be lower for this variant.

iv) An other important fact is associated to the density of nucleus. A dense distribution of nucleus of a unique variant would favor the growth of such variant and would serve as obstacle for the other variants. The advantages for the TWSME would be of the order of magnitude of the nucleation energy. The nucleation energy in turn is related to the hysteresis width, which is in the range of a few degrees.

These remarks are consistent with the fact that the TWSME appears together with the complex defects [22], which correspond to the basal plane type defects optically observed [13]. Such type defects have been also observed in samples cycled at lower temperatures [23]. In this later case the stabilization processes would hardly occur and the TWSME is more likely related to the complex defects.

\section{ACKNOWLEDGMENTS:}

The work was partially supported by the DGICYT of Spain (sabatic program), The Commission of European Communities under contract ALAMED CI1*-CT91-093 and the CONICET of Argentina.

\section{REFERENCES:}

[1] Schroeder T.A. and Wayman C.M., Scripta metall., 11 (1977) 225- 230

[2] Perkins J. and Sponholtz R.O., Metall. Trans., 15A (1984) 313-321.

[3] Jean R., Lai H.J. and Cheuh S.C., Proc. Int. Conference on Martensitic Transformations (Monterey, Editors: Wayman C.M. and Perkins J.) 1992, 1119-1123.

[4] Rapacioli R., Torra V., Cesari E., Guilemany J.M. and Miguel J.R., Scripta metall., 22 (1988) 261-264

[5] Pons J., Sade M., Lovey F.C. and Cesari E., Proc. Int. Conference on Martensitic Transformations (Monterey, Editors: Wayman C.M. and Perkins J.) 1992, 1113-1118. Ibid Mat. Trans. JM, 34 (1993) 888-894.

[6] Contardo L. and Guenin G., Acta metall. mater., 38 (1990) 1267-1272.

[7] Stalmans R., Van Humbeeck J. and Delaey L., Acta metall. mater., 40 (1992) 501-511. Ibid, 40 (1992) 2921-2931.

[8] Sade M., Hazarabedian A., Uribarri A. and Lovey F.C., Proc. Int. Conference on Phase Transformations (G.W. Lorimer, ed., The Institute of Metals, Cambridge), 1987, 279-283.

[9] Cesari E., Picornell C. and Sade M., Proc. Int. Conference on Martensitic Transformations (Monterey, Editors: Wayman C.M. and Perkins J.) 1992, 1107-1112. Picornell C., Sade M. and Cesari E., Metall. Trans. A, 25 (1994) 1-9.

[10] Rios-Jara D. and Guenin G., Acta metall., 35 (1987) 109-119.

[11] Sade M., Uribarri A. and Lovey F.C., Phil. Mag.A, 55 (1987) 455- 461.

[12] Lovey F.C., Sade M., Torra V. and Amengual A., Proc. Int. Conference on Martensitic Transformations (Monterey, Editors: Wayman C.M. and Perkins J.) 1992, 365-370.

[13] Cingolani E., Ahlers M. and Sade M., to be published.

[14] Sade M., Rapacioli R. and Ahlers M., Acta metall., 33 (1985) 487-497.

[15] Cuniberi A., Romero R. and Ahlers M., Scripta metall. mater., 26 (1992) 495-500.

[16] Romero R., Lovey F.C. and Ahlers M., Phil. Mag. A, 58 (1988) 881-903.

[17] Olson G.B. and Cohen M., Proc. Int. Conference on Solid-Solid Phase Transformations, Pittsburg, (1981) 1145-1164.

[18] Eshelby J.D., Proc. Roy. Soc., 241 (1957) 376-396.

[19] Rodriguez, P.L. and Lovey F.C., unpublished.

[20] Rodriguez P.L., Lovey F.C., Guenin G., Pelegrina J.L., Sade M. and Morin M., Acta metall. mater., 41 (1993) 3307-3310.

[21] Lovey F.C., Hazarabedian A. and Garces J.E., Acta metall., 37 (1989) 2321-2327.

[22] Hazarabedian A., Master Thesis, Instituto Balseiro, Bariloche, Argentina (1986).

[23] Sade M. and Ahlers M., 19 (1985) 425-430. 